Pacific Journal of Mathematics

AN INDEX CHARACTERIZATION OF THE CATENOID AND
INDEX BOUNDS FOR MINIMAL SURFACES IN R 


\title{
AN INDEX CHARACTERIZATION OF THE CATENOID AND INDEX BOUNDS FOR MINIMAL SURFACES IN $\mathbf{R}^{4}$
}

\author{
Shiu-Yuen Cheng AND Johan Tysk
}

\begin{abstract}
The index of a minimal surface is defined to be the number of negative eigenvalues of the operator corresponding to second variation of area. In the present paper, we characterize the catenoid as the only complete oriented minimal surface in $R^{3}$ of index one with embedded ends. We also obtain upper bounds for the index of minimal surfaces in $\mathbf{R}^{4}$, in terms of the total curvature of the surface.
\end{abstract}

1. Introduction. The index of a minimal surface measures how far the surface is from being stable, with index equal to zero corresponding to stability. For noncompact surfaces $M$, one defines the index as the limit of the indices of an exhausting sequence of compact domains $D$ in $M$. The index of a domain $D$ is the number of negative eigenvalues of the Dirichlet eigenvalue problem on $D$ for the operator corresponding to second variation of area. For oriented minimal surfaces in $\mathbf{R}^{3}$, this eigenvalue problem has the form

$$
(\Delta-2 K) \phi+\lambda \phi=0 \quad \text { on } D,\left.\quad \phi\right|_{\partial D}=0,
$$

where $K$ is the Gaussian curvature of $M$, and $\Delta$ the Laplace-Beltrami operator on $M$.

D. Fischer-Colbrie and R. Schoen [FC-S], showed that the only complete oriented minimal surface in $\mathbf{R}^{3}$ of index zero is a plane. Fischer-Colbrie [FC], then showed that the index of a complete oriented minimal surface in $\mathbf{R}^{3}$ is finite if and only if the total curvature of the surface is finite. Her results, however, give no explicit information on how the index varies with the total curvature. In [T1], it is shown, using heat-kernel techniques, that the index is bounded by an explicit constant times the total curvature.

In $\S 2$ of this paper, we obtain a weaker type of bound from below for the index of minimal surfaces in $\mathbf{R}^{3}$. We show that all oriented complete minimal surfaces with embedded ends, except the plane and the catenoid, have index at least two. This yields as a corollary the characterization of the catenoid as the only complete oriented minimal surface of index one, with embedded ends. 
Many open questions naturally arise in this context. Can any minimal surface in $\mathbf{R}^{3}$, apart from the plane and the catenoid, be characterized by its index? Can one bound the index from below by a constant multiple of the total curvature? What are the indices of the families of minimal surfaces constructed by D. Hoffman and W. Meeks, see [H-M1] and [H-M2]? Since the surfaces constructed by Hoffman and Meeks form one-parameter families, the different surfaces in these families cannot be characterized by their indices. Still, if the surface is embedded, can we determine its topology from its index?

Before considering the upper bound for the index of minimal surfaces in $\mathbf{R}^{4}$ derived in the last section of the present paper, we review some related results. Consider the operator

$$
\Delta+W
$$

on $\mathbf{R}^{n}, n \geq 3$, where $\Delta$ is the Laplace operator and $W$ a potential. According to a theorem by P. Li and S.-T. Yau [L-Y], the number of negative eigenvalues of this operator is bounded by a constant multiple of the integral of $|W|^{n / 2}$. Their argument can be carried out on any manifold on which a Sobolev inequality holds. Now, the second variation operator for an oriented minimal submanifold $M^{n}$ of $\mathbf{R}^{n+1}$, has the form

$$
\Delta+|A|^{2}
$$

where $|A|^{2}$ is the square of the length of the second fundamental form of $M^{n}$ as a submanifold of $\mathbf{R}^{n+1}$, and $\Delta$ is the Laplace-Beltrami operator on $M^{n}$. Since the Sobolev inequality holds for minimal submanifolds of Euclidean space, see [M-S], we obtain for $n \geq 3$,

$$
\operatorname{index}\left(M^{n}\right) \leq C(n) \int_{M^{n}}|A|^{n},
$$

which was observed in [T2]. P. Bérard and G. Besson [B-B], showed that this estimate holds for minimal submanifolds of dimension at least three and of arbitrary codimension in Euclidean space.

The result, quoted above, by P. Li and S.-T. Yau does not hold in $\mathbf{R}^{2}$ : if the potential $W$ is nonnegative and positive somewhere, then $\Delta+W$ on $\mathbf{R}^{2}$ has at least one negative eigenvalue. This is seen by using the fact that the Dirichlet integral is conformally invariant in dimension two. The upper bound for the index of minimal surfaces in $\mathbf{R}^{3}$ given in [T1], therefore had to be obtained by completely different methods. Note that this index estimate does not contradict the failure of the theorem of P. Li and S.-T. Yau in dimension two, since if $M^{2}$ is an 
oriented minimal surface in $\mathbf{R}^{3}$ with

$$
\int_{M}(-K)<4 \pi
$$

then $M^{2}$ is a plane.

As mentioned above, in the last section of this paper, we show that the index of a minimal surface in $\mathbf{R}^{4}$ is bounded by a constant multiple of the total curvature. Our proof uses the fact that the Grassmannian $G_{2,4}$ of two-planes in four-space splits as a product of two spheres, and therefore does not immediately generalize to minimal surfaces in Euclidean spaces of dimension at least five. We therefore leave the following question open: for minimal surfaces $M^{2}$ in $\mathbf{R}^{n}, n \geq 5$, is the index of $M^{2}$ bounded by a constant multiple, depending only on $n$, of the total curvature?

After the completion of this paper we learned that F. J. Lopez and A. Ros [L-R] have shown that the catenoid and the Enneper surface are the only complete oriented minimal surfaces in $\mathbf{R}^{3}$ of index one. The method of proof we used to characterize the catenoid as the only complete oriented minimal surface of index one with embedded ends is, however, different from the argument offered by F. J. Lopez and A. Ros.

2. A characterization of the catenoid by its index. We derive a lower bound for the index of minimal surfaces in $\mathbf{R}^{3}$ as a consequence of the characterization, due to $\mathbf{R}$. Schoen [S], of the catenoid as the only complete minimal surface in $\mathbf{R}^{\mathbf{3}}$ with two regular embedded ends and of the eigenvalue estimate for Riemann surfaces by P. C. Yang and S.-T. Yau, see [Y-Y]. The characterization of the catenoid by its index follows from this lower bound.

THEOREM 1. Let $M$ be a complete oriented minimal surface in $\mathbf{R}^{3}$ which has embedded ends, and which is neither a plane nor a catenoid. Then

$$
\operatorname{index}(M) \geq 2
$$

Proof. If $M$ has infinite total curvature then the index of $M$ is infinite by [FC], so it is enough to show the lower bound for minimal surfaces with finite total curvature. From the classical results of $\mathbf{R}$. Osserman [O], it then follows that the Gauss map $G$ of $M$ has a holomorphic extension to a Riemann surface compactification $\bar{M}$ of $M$. When $M$ is not a plane, we therefore have a non-constant holomorphic 
mapping

$$
G: \bar{M} \rightarrow S^{2} .
$$

Instead of considering the second variation operator

$$
\Delta-2 K
$$

on $M$, we can consider the corresponding operator on $\bar{M}$ with respect to the pull-back metric $G^{*}\left(d s_{S^{2}}^{2}\right)$ of the metric $d s_{S^{2}}^{2}$ on the sphere. The metric $G^{*}\left(d s_{S^{2}}^{2}\right)$ satisfies $G^{*}\left(d s_{S^{2}}^{2}\right)=(-K) d s_{M}^{2}$ and might have isolated singular points. Note however, that we can still speak of the eigenvalues of the Laplace operator with respect to the metric $G^{*}\left(d s_{S^{2}}^{2}\right)$ since a finite set of points does not affect the variational characterization of the eigenvalues. The Laplace operator $\Delta_{\bar{M}}$ on $\left(\bar{M}, G^{*}\left(d s_{S^{2}}^{2}\right)\right)$ satisfies

$$
\Delta=(-K) \Delta_{\bar{M}}
$$

Hence, the operator $\Delta-2 K$ on $M$ takes the form

$$
(-K)\left(\Delta_{\bar{M}}+2\right)
$$

on $\left(\bar{M}, G^{*}\left(d s_{S^{2}}^{2}\right)\right)$. The index of $M$ is therefore equal to the number of negative eigenvalues of $\Delta_{\bar{M}}+2$ on $\bar{M}$, or equivalently, the number of eigenvalues of $\Delta_{\bar{M}}$ that are strictly less than two.

The number of sheets of the branched covering

$$
G: \bar{M} \rightarrow S^{2}
$$

is

$$
k=\frac{1}{4 \pi} \int_{M}(-K) d V
$$

We can then directly apply Theorem 1 of P. C. Yang and S.-T. Yau in [Y-Y] to conclude that the first nonzero eigenvalue $\lambda_{1}$ of $\bar{M}$ satisfies

$$
\lambda_{1} \leq \frac{8 \pi(g+1)}{4 \pi k}=2 \frac{g+1}{k},
$$

where $g$ is the genus of $\bar{M}$. Now, for immersed oriented minimal surfaces in $\mathbf{R}^{3}$ we have, see [O],

$$
k \geq g+r-1
$$

where $r$ is the number of ends of $M$. By using the maximum principle on the coordinate functions of the ambient Euclidean space, we find that a minimal surface in $\mathbf{R}^{3}$ of finite total curvature with one embedded end has to be a plane. Furthermore, if $r=2$ and the ends of $M$ are embedded, we know that $M$ is a catenoid by $\mathbf{R}$. Schoen [S]. Since 
we are excluding the plane and the catenoid from over index estimate, we can assume that $r \geq 3$, so that

$$
k \geq g+2 \text {. }
$$

By estimate (1) above, we then have

$$
\lambda_{1} \leq 2 \frac{g+1}{k} \leq 2 \frac{g+1}{g+2}<2 .
$$

We therefore have two eigenvalues of $\Delta_{\bar{M}}$ that are strictly less than two: $\lambda_{0}=0$ and $\lambda_{1}$. Since the index of $M$ is equal to the number of eigenvalues of $\Delta_{\bar{M}}$ that are strictly less than two, this completes our proof.

In the case of the catenoid, the Gauss map is a diffeomorphism from $M$ to the sphere minus two points. The index of the catenoid is therefore equal to the number of eigenvalues of the sphere that are strictly less than two, i.e., the index of the catenoid is one. Hence, our index estimate yields the following immediate corollary.

COROLLARY 1. The only complete oriented minimal surface in $\mathbf{R}^{3}$ of index one with embedded ends is the catenoid.

3. Index bounds for minimal surfaces in $\mathbf{R}^{4}$. In this section we obtain upper bounds for the index of oriented minimal surfaces in $\mathbf{R}^{4}$. The index is bounded above by a constant multiple of the total curvature.

THEOREM 2. Let $M^{2} \rightarrow \mathbf{R}^{4}$ be an immersed complete oriented minimal surface. Then,

$$
\operatorname{index}(M) \leq 12.72\left(\frac{1}{2 \pi} \int_{M}(-K)\right) .
$$

REMARK 1. The analogous statement holds for minimal surfaces in manifolds covered by $\mathbf{R}^{4}$.

REMARK 2. For a non-orientable minimal surface we can bound the index by the index of its two-sheeted oriented cover. Hence, for nonorientable surfaces the estimate above holds if we double the given multiple of the total curvature.

REMARK 3. It is not possible to bound the index from below by a multiple of the total curvature, since if $M^{2}$ in $\mathbf{R}^{4} \simeq \mathbf{C} \times \mathbf{C}$ is a holomorphic subvariety, then $M^{2}$ is always stable.

Proof. Let $N(M)$ denote the normal bundle of $M$ and let $E$ be a smooth compactly supported section of this bundle. The second 
derivative of the area of $M$ under the variation corresponding to $E$ is given by

$$
\left.\frac{d^{2} A}{d s^{2}}\right|_{s=0}=\int_{M}\left(\langle\nabla E, \nabla E\rangle-\left\langle B^{t}(E), B^{t}(E)\right\rangle\right) d V
$$

where $d V$ denotes the volume form of $M$. For the derivation of this formula, see [L]. Here

$$
\langle\nabla E, \nabla E\rangle=\sum_{i=1}^{2}\left\langle\nabla_{e_{i}} E, \nabla_{e_{i}} E\right\rangle,
$$

for orthonormal vectors $e_{i} \in T(M), i=1,2$. The connection is defined by

$$
\nabla_{e_{i}} E=\left(\nabla_{e_{i}}^{\mathbf{R}^{4}} E\right)^{N},
$$

where $\nabla^{\mathbf{R}^{4}}$ is the standard connection on $\mathbf{R}^{4}$ and ( ) ${ }^{N}$ denotes projection onto $N(M)$. Also,

$$
B^{t}: N_{p}(M) \rightarrow T_{p}(M) \otimes T_{p}(M),
$$

is the transpose of the second fundamental form

$$
B: T_{p}(M) \otimes T_{p}(M) \rightarrow N_{p}(M),
$$

defined by

$$
B_{X, Y}=\left(\nabla_{X}^{\mathbf{R}^{4}} Y\right)^{N}
$$

Hence,

$$
\left\langle B^{t}(E), B^{t}(E)\right\rangle=\sum_{i, j=1}^{2}\left\langle E, B_{e_{i}, e_{j}}\right\rangle^{2} \leq|E|^{2}|B|^{2}
$$

so that

$$
\left.\frac{d^{2} A}{d s^{2}}\right|_{s=0} \geq \int_{M}\left(\langle\nabla E, \nabla E\rangle-|B|^{2}|E|^{2}\right) d V .
$$

Consider the Gauss map

$$
G: M \rightarrow G_{2,4},
$$

where $G_{2,4}$ is the Grassmannian of oriented two-planes in $\mathbf{R}^{4}$. We can assume that $M$ has finite total curvature, since otherwise our index estimate is trivially satisfied. For minimal surfaces of finite total curvature the Gauss map extends as an anti-holomorphic mapping to a Riemann surface compactification $\bar{M}$ of $M$, see [C-O], and the pullback metric satisfies

$$
G^{*}\left(d s_{G_{2,4}}^{2}\right)=(-K) d s_{M}^{2}
$$


Recall that for minimal surfaces $|B|^{2}=-2 K$. Hence,

$$
\begin{aligned}
\int_{M} & \left(\langle\nabla E, \nabla E\rangle-|B|^{2}|E|^{2}\right) d V \\
& =\int_{M}\left(\sum_{i=1}^{2}\left\langle\nabla_{e_{i}} E, \nabla_{e_{i}} E\right\rangle-2|E|^{2}(-K)\right) d V \\
& =\int_{M}\left(\sum_{i=1}^{2}\left\langle\nabla_{(-K)^{-1 / 2} e_{i}} E, \nabla_{(-K)^{-1 / 2} e_{i}} E\right\rangle-2|E|^{2}\right)(-K) d V \\
& =\int_{\bar{M}}\left(\langle\bar{\nabla} E, \bar{\nabla} E\rangle-2|E|^{2}\right) d \bar{V},
\end{aligned}
$$

where $\bar{\nabla}$ is the gradient on $N(\bar{M})$ induced by the metric $(-K) d s_{M}^{2}$ on $\bar{M}$, and $d \bar{V}$ is the corresponding volume form. The index of $M$ can therefore be estimated by the number of negative eigenvalues of

$$
\bar{\Delta}+2
$$

on $N(\bar{M})$, where $\bar{\Delta}$ denotes the Laplace operator on $N(\bar{M})$ induced by the metric $(-K) d s_{M}^{2}$ on $\bar{M}$. Let $\left\{\bar{\lambda}_{i}\right\}_{i=0}^{\infty}$ be the eigenvalues of $\bar{\Delta}$, and let $\left\{\lambda_{i}\right\}_{i=0}^{\infty}$ be the eigenvalues of the Laplace-Beltrami operator on functions on $\bar{M}$. According to a theorem by H. Hess, S. Schrader and D. A. Uhlenbrock in [H-S-U],

$$
\sum_{i=0}^{\infty} e^{-\bar{\lambda}_{i} t} \leq \operatorname{rank}(N(\bar{M})) \sum_{i=0}^{\infty} e^{-\lambda_{i} t}=2 \sum_{i=0}^{\infty} e^{-\lambda_{t} t},
$$

for all $t \geq 0$. Their theorem is stated for the case of smooth Hermitian vector bundles. However, it is easily seen to apply to our case by considering the complexified bundle $N(\bar{M}) \otimes \mathbf{C}$ and extending the inner product on $N(\bar{M})$ to a Hermitian inner product on $N(\bar{M}) \otimes \mathbf{C}$. We then extend $\bar{\nabla}$ to a complex linear connection which is Hermitian with respect to the metric on $N(\bar{M}) \otimes \mathbf{C}$. Furthermore, the connection $\bar{\nabla}$ on $N(\bar{M}) \otimes \mathrm{C}$ has singularities at points where $K=0$, but since there are only finitely many such points, a straightforward approximation argument makes it clear that the inequality in [H-S-U] also holds in our case.

Now,

$$
G_{2,4}=S_{1} \times S_{2}
$$

where $S_{1}$ and $S_{2}$ are spheres of radius $1 / \sqrt{2}$, see for example [W]. Let

$$
g_{j}=\pi_{j} \circ G, \quad j=1,2,
$$


be the projection of the Gauss map onto the $j$ th factor of $G_{2,4}$. Since $G$ is anti-holomorphic, the maps $g_{j}$ are conformal. Consider the metrics

$$
2 g_{j}^{*}\left(d s_{s_{j}}^{2}\right)=2 e^{\rho_{J}} d s_{M}^{2},
$$

where $\rho_{j}, j=1,2$, are functions with values in $[-\infty, \infty)$. Let

$$
\left\{\lambda_{i}^{j}\right\}_{i=0}^{\infty}, \quad j=1,2,
$$

be the eigenvalues of the Laplace-Beltrami operator on $\left(\bar{M}, 2 e^{\rho_{j}} d s_{M}^{2}\right)$. Then, we claim that

$$
\sum_{i=0}^{\infty} e^{-\lambda_{i} t} \leq \sum_{j=1}^{2} \sum_{i=0}^{\infty} e^{-\lambda_{i}^{j} t}
$$

To see this, let $N(\mu)$ denote the number of eigenvalues of $\left(\bar{M}, G^{*}\left(d s_{G_{24}}^{2}\right)\right)$ that are strictly less than $\mu$ and let $N_{j}(\mu), j=1,2$, denote the eigenvalues of $\left(\bar{M}, 2 g_{j}^{*}\left(d s_{S}^{2}\right)\right)$ that are strictly less than $\mu$. Now, note that $N(\mu)$ is bounded by the largest dimension of a linear space $L$ of piecewise differentiable functions on which the quadratic form

$$
I(u, v)=\int_{\bar{M}} \nabla u \cdot \nabla v-\mu \int_{\bar{M}} u v(-K) d V
$$

is negative definite. Since the Dirichlet integral is conformally invariant in dimension two we deliberately suppress the volume form used in the first integral. Let $\left\{u_{1}, \ldots, u_{m}\right\}$ be a basis for $L$. Set, for $1 \leq i$, $j \leq m$,

$$
\begin{aligned}
a_{i j} & =\int_{\bar{M}} \nabla u_{i} \cdot \nabla u_{j}, \\
b_{i j} & =\int_{\bar{M}} u_{i} u_{j} e^{\rho_{1}} d V, \\
c_{i j} & =\int_{\bar{M}} u_{i} u_{j} e^{\rho_{2}} d V .
\end{aligned}
$$

On $L$, we have in the sense of matrices,

$$
\left(a_{i j}\right)-\mu\left(b_{i j}\right)-\mu\left(c_{i j}\right)<0
$$

Choose a basis for $L$, by abuse of notation denoted $\left\{u_{1}, \ldots, u_{m}\right\}$, such that $\left(b_{i j}\right)$ is the identity matrix and $\left(c_{i j}\right)$ is diagonalized. We order the basis vectors so that $c_{i i} \leq 1$, for $i \leq l$, and $c_{i i}>1$, for $i>l$, for some $l, 0 \leq l \leq m$. Using the properties of $\left(b_{i j}\right)$ and $\left(c_{i j}\right)$, we find that

$$
\left(a_{i j}\right)-2 \mu\left(b_{i j}\right)<0
$$


on $\operatorname{span}\left\{u_{1}, \ldots, u_{l}\right\}$, and

$$
\left(a_{i j}\right)-2 \mu\left(c_{i j}\right)<0,
$$

on $\operatorname{span}\left\{u_{l+1}, \ldots, u_{m}\right\}$. The dimensions of these spaces are bounded by $N_{1}(\mu)$ and $N_{2}(\mu)$, respectively. Hence,

$$
N(\mu) \leq N_{1}(\mu)+N_{2}(\mu)
$$

for any $\mu$, and our claim follows.

According to the estimate of traces of heat kernels in [T1],

$$
\sum_{i=0}^{\infty} e^{-\lambda_{i}^{\prime} t} \leq\left(\operatorname{deg} g_{j}\right) \sum_{i=0}^{\infty} e^{-\mu_{i} t}, \quad j=1,2,
$$

where $\left\{\mu_{i}\right\}_{i=0}^{\infty}$ are the eigenvalues of the standard $S^{2}$. Hence, in conclusion,

$$
\begin{aligned}
\sum_{i=0}^{\infty} e^{-\bar{\lambda}_{t} t} & \leq 2 \sum_{i=0}^{\infty} e^{-\lambda_{t} t} \leq 2 \sum_{j=1}^{2} \sum_{i=0}^{\infty} e^{-\lambda_{i}^{j} t} \\
& \leq 2\left(\operatorname{deg} g_{1}+\operatorname{deg} g_{2}\right) \sum_{i=0}^{\infty} e^{-\mu_{i} t}
\end{aligned}
$$

and therefore,

$$
\begin{aligned}
(\operatorname{index}(M)) e^{-2 t} & \leq \sum_{\lambda_{i}<2} e^{-\bar{\lambda}_{t} t} \leq \sum_{i=0}^{\infty} e^{-\bar{\lambda}_{i} t} \\
& \leq 2\left(\operatorname{deg} g_{1}+\operatorname{deg} g_{2}\right) \sum_{i=0}^{\infty} e^{-\mu_{i} t},
\end{aligned}
$$

so that

$$
\operatorname{index}(M) \leq 2\left(\operatorname{deg} g_{1}+\operatorname{deg} g_{2}\right) e^{2 t} \sum_{i=0}^{\infty} e^{-\mu_{i} t}
$$

Now, since the area of the image of the Gauss map is

$$
\int_{M}(-K)
$$

and the area of the spheres $S_{1}$ and $S_{2}$ is $2 \pi$, we have

$$
\operatorname{deg} g_{1}+\operatorname{deg} g_{2}=\frac{1}{2 \pi} \int_{M}(-K) \text {. }
$$

The $i$ th distinct eigenvalue of $S^{2}$ is known to be $i(i+1)$, with multiplicity $2 i+1$. Using this, we find that $t \approx 0.426$ gives the smallest 
possible value of 6.36 of the coefficient of $2\left(\operatorname{deg} g_{1}+\operatorname{deg} g_{2}\right)$, thus proving our theorem.

\section{REFERENCES}

[B-B] P. Bérard and G. Besson, Number of bound states and estimates on some geometric invariants, preprint.

[C-O] S. S. Chern and R. Osserman, Complete minimal surfaces in Euclidean nspace, J. d'Analyse Math., 19 (1967), 15-34.

[FC] D. Fischer-Colbrie, On complete minimal surfaces with finite Morse index in three-manifolds, Invent. Math., 82 (1985), 121-132.

[FC-S] D. Fischer-Colbrie and R. Schoen, The structure of complete stable minimal surfaces in three-manifolds of non-negative scalar curvature, Comm. Pure Appl. Math., 33 (1980), 199-211.

[H-M1] D. Hoffman and W. H. Meeks III, A complete embedded minimal surface in $R^{3}$ with genus one and three ends, J. Diff. Geom., 21 (1985), 109-127.

[H-M2] One-parameter families of embedded minimal surfaces, in preparation.

[H-S-U] H. Hess, R. Schrader, and D. A. Uhlenbrock, Kato's inequality and the spectral distribution of Laplacians on compact Riemannian manifolds, $\mathbf{J}$. Diff. Geom., 15 (1980), 27-37.

[L] H. B. Lawson, Minimal varieties in real and complex geometry, Les Presses de l'Université de Montréal.

[L-R] F. J. Lopez and A. Ros, Complete minimal surfaces with index one and stable constant mean curvature surfaces, preprint.

[L-Y] P. Li and S.-T. Yau, On the Schrödinger equation and the eigenvalue problem, Commun. Math. Phys., 88 (1983), 309-318.

[M-S] J. H. Michael and L. M. Simon, Sobolev and mean value inequalities on generalized submanifolds of $\mathbf{R}^{n}$, Commun. Pure Appl. Math., 26 (1973), 361-379.

[O] R. Osserman, Global properties of minimal surfaces in $E^{3}$ and $E^{n}$, Ann. of Math., 80 (1964), 340-364.

[S] R. Schoen, Uniqueness, symmetry and embeddedness of minimal surfaces, J. Diff. Geom., 18 (1983), 791-809.

[T1] J. Tysk, Eigenvalue estimates with applications to minimal surfaces, Pacific J. Math., 128 (1987), 361-366.

[T2] _ Finiteness of index and total scalar curvature for minimal hypersurfaces, preprint.

[W] P. Wintgen, On the total curvature of surfaces in $E^{4}$, Colloq. Math., 39 (1978), 289-296.

[Y-Y] P. C. Yang and S.-T. Yau, Eigenvalues of the Laplacian of compact Riemann surfaces and minimal submanifolds, Ann. Sci. Ec. Norm. Super. Pisa, 7 (1980), 55-63.

Received June 2, 1987.

UNIVERSITY OF CALIFORNIA

LOS ANGeles, CA 90024-1555

AND

COLUMBia UNIVERSITY

NEW YORK, NY 10027 


\section{EDITORS}

\author{
V. S. VARADARAJAN \\ (Managing Editor) \\ University of California \\ Los Angeles, CA 90024 \\ HeRBert Clemens \\ University of Utah \\ Salt Lake City, UT 84112 \\ R. FINN \\ Stanford University \\ Stanford, CA 94305
}

\author{
HERMANN FLASCHKA \\ University of Arizona \\ Tucson, AZ 85721
}

RAMESh A. GANGOLLI University of Washington Seattle, WA 98195

VAUGHAN F. R. JONES University of California

Berkeley, CA 94720
ROBION KIRBY

University of California

Berkeley, CA 94720

C. C. MOORE

University of California

Berkeley, CA 94720

HAROLD STARK

University of California, San Diego

La Jolla, CA 92093

\section{ASSOCIATE EDITORS}
R. ARENS
E. F. BECKENBACH
B. H. NEUMANN
F. WOLF
K. YOSHIDA

(1906-1982)

\section{SUPPORTING INSTITUTIONS}

UNIVERSITY OF ARIZONA
UNIVERSITY OF BRITISH COLUMBIA
CALIFORNIA INSTITUTE OF TECHNOLOGY
UNIVERSITY OF CALIFORNIA
MONTANA STATE UNIVERSITY
UNIVERSITY OF NEVADA, RENO
NEW MEXICO STATE UNIVERSITY
OREGON STATE UNIVERSITY

UNIVERSITY OF ARIZONA

UNIVERSITY OF BRITISH COLUMBIA

UNIVERSITY OF CALIFORNIA

MONTANA STATE UNIVERSITY

NEW MEXICO STATE UNIVERSITY

OREGON STATE UNIVERSITY

\author{
UNIVERSITY OF OREGON \\ UNIVERSITY OF SOUTHERN CALIFORNIA \\ STANFORD UNIVERSITY \\ UNIVERSITY OF HAWAII \\ UNIVERSITY OF TOKYO \\ UNIVERSITY OF UTAH \\ WASHINGTON STATE UNIVERSITY \\ UNIVERSITY OF WASHINGTON
}

The Supporting Institutions listed above contribute to the cost of publication of this Journal, but they are not owners or publishers and have no responsibility for its content or policies.

Mathematical papers intended for publication in the Pacific Journal of Mathematics should be in typed form or offset-reproduced (not dittoed), double spaced with large margins. Please do not use built up fractions in the text of the manuscript. However, you may use them in the displayed equations. Underline Greek letters in red, German in green, and script in blue. The first paragraph must be capable of being used separately as a synopsis of the entire paper. In particular it should contain no bibliographic references. Please propose a heading for the odd numbered pages of less than 35 characters. Manuscripts, in triplicate, may be sent to any one of the editors. Please classify according to the scheme of Math. Reviews, Index to Vol. 39. Supply name and address of author to whom proofs should be sent. All other communications should be addressed to the managing editor, or Elaine Barth, University of California, Los Angeles, California 90024.

There are page-charges associated with articles appearing in the Pacific Journal of Mathematics. These charges are expected to be paid by the author's University, Government Agency or Company. If the author or authors do not have access to such Institutional support these charges are waived. Single authors will receive 50 free reprints; joint authors will receive a total of 100 free reprints. Additional copies may be obtained at cost in multiples of 50 .

The Pacific Journal of Mathematics is issued monthly as of January 1966. Regular subscription rate: $\$ 190.00$ a year (5 Vols., 10 issues). Special rate: $\$ 95.00$ a year to individual members of supporting institutions.

Subscriptions, orders for numbers issued in the last three calendar years, and changes of address should be sent to Pacific Journal of Mathematics, P.O. Box 969, Carmel Valley, CA 93924, U.S.A. Old back numbers obtainable from Kraus Periodicals Co., Route 100, Millwood, NY 10546.

The Pacific Journal of Mathematics at P.O. Box 969, Carmel Valley, CA 93924 (ISSN 0030-8730) publishes 5 volumes per year. Application to mail at Second-class postage rates is pending at Carmel Valley, California, and additional mailing offices. Postmaster: send address changes to Pacific Journal of Mathematics, P.O. Box 969, Carmel Valley, CA 93924.

\section{PUBLISHED BY PACIFIC JOURNAL OF MATHEMATICS, A NON-PROFIT CORPORATION} Copyright (c) 1988 by Pacific Journal of Mathematics 


\section{Pacific Journal of Mathematics}

Vol. 134, No. $2 \quad$ June, 1988

P. D. Allenby and M. Sears, Extension of flows via discontinuous functions

Arthur William Apter and Moti Gitik, Some results on Specker's

problem

Shiu-Yuen Cheng and Johan Tysk, An index characterization of the catenoid and index bounds for minimal surfaces in $\mathbf{R}^{4}$

Mikihiro Hayashi and Mitsuru Nakai, Point separation by bounded analytic functions of a covering Riemann surface

Charles Philip Lanski, Differential identities, Lie ideals, and Posner's theorems

Erich Miersemann, Asymptotic expansion at a corner for the capillary problem

Dietrich W. Paul, Theory of bounded groups and their bounded cohomology

Ibrahim Salama, Topological entropy and recurrence of countable chains

Zbigniew Slodkowski, Pseudoconvex classes of functions. I. Pseudoconcave

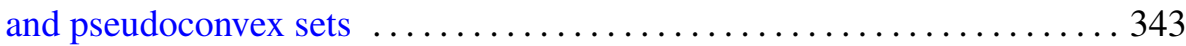

Alfons Van Daele, $K$-theory for graded Banach algebras. II . . . . . . . . . . 377

Edwin Wolf, Functions in $R^{2}(E)$ and points of the fine interior 393 